\title{
Dosis de radiación ultravioleta en escolares mexicanos
}

\author{
Juan Pablo Castanedo-Cázares, MC, (1) Verónica Lepe, MC, ${ }^{(1)}$ \\ Antonio Gordillo-Moscoso, MC, M en $C^{(2)}$ Benjamín Moncada, MC.(1)
}

\begin{abstract}
Castanedo-Cázares JP, LepeV, Gordillo-Moscoso A, Moncada B. Dosis de radiación ultravioleta en escolares mexicanos. Salud Publica Mex 2003;45:439-444. El texto completo en inglés de este artículo está disponible en: http://www.insp.mx/salud/index.html
\end{abstract}

\section{Resumen}

Objetivo. Determinar la dosis de radiación ultravioleta que reciben los niños y adolescentes durante su asistencia a la escuela primaria, secundaria o preparatoria en la ciudad de San Luis Potosí. Material y métodos. Estudio de cohorte hecho en la ciudad de San Luis Potosí entre mayo de 2001 y abril de 2002. Se evaluaron 80 escolares escogidos al azar de 6 a 19 años de edad, de uno u otro sexo. La dosis se cuantificó durante todo un ciclo escolar mediante el registro del tiempo de exposición solar para cada uno de los alumnos, de modo simultáneo al monitoreo de radiación ultravio leta, para lo cual se utilizaron equipos de radiometría terrestre. Se excluyeron las vacaciones y los fines de semana. Los datos se analizaron en forma univariada y comparativamente, por sexo y meses de exposición; además, se buscó un modelo de regresión para explicar la dosis de exposición solar. Resultados. La dosis promedio ajustada al espectro de eritematógeno acción fue de $16456 \mathrm{~J} / \mathrm{m}^{2} /$ año. Hubo diferencias por género: 14264 $\mathrm{J} / \mathrm{m}^{2} /$ año/mujeres vs. $18648 \mathrm{~J} / \mathrm{m}^{2} /$ año/hombres (Kolmogorov-Smirnof, $p=0.003$ ). No hubo diferencias significativas entre grupos, pero sí entre los meses de exposición (Kruskall-W allis, $p=<0.0001$ ). U tilizando la técnica estadística de modelos lineales generalizados, y mediante técnica escalonada y calificación del índice de Akaike se escogió el mejor modelo que explica la dosis de radiación según el

\author{
Castanedo-Cázares JP, LepeV, \\ Gordillo-Moscoso A, Moncada B. \\ Ultraviolet radiation doses of Mexican schoolchildren. \\ Salud Publica Mex 2003;45:439-444. \\ The English version of this paper \\ is available at: http://www.insp.mx/salud/index.html
}

\begin{abstract}
A bstract
Objective. To determine the ultraviolet radiation dose received by children and adolescents at elementary, middle, or high school. Material and Methods. A cohort study was conducted in the City of San Luis Potosí between May 2001 and A pril 2002. The study population consisted of 80 school male and female children, selected at rando $m$, aged 6 to 19 years of age.The dose was quantitated during an entire schoolyear by recording the time each student was exposed to sunlight. Ultraviolet radiation was simultaneo usly monitored with terrestrial radiometry equipment. Holidays and weekends were excluded. Data were analyzed using univariate analysis and comparative analysis by sex and months of exposure. A regression model was fit to explain the dose of solar exposure. Results. The erythemally weighted UV dose for Mexican schoolchildren averaged $16456 \mathrm{~J} /$ $\mathrm{m}^{2} /$ year. D ifferences by gender were found: $14,264 \mathrm{~J} / \mathrm{m}^{2} /$ year in females vs. $18,648 \mathrm{~J} / \mathrm{m}^{2} /$ year in males (KolmogorovSmirnov, $p=0.003$ ). No significant differences were found among groups. Significant differences were found among months of exposure (Kruskal-W allis, $p=<0.0001$ ). Stepwise regression models were fit to find the best model, using generalized linear modeling and the A kaike information criterion, to explain the radiation dose according to month of exposure; the final equation was $587.20+438.45$ (gender) +500.16 (month)- 49.65 (month ${ }^{2}$ ). The results showed higher
\end{abstract}

(1) Departamento de Dermatología. Hospital Central D r. Ignacio Morones Prieto. Facultad de Medicina. Universidad Autónoma de San Luis Potosí, San Luis Potosí, México.

(2) Hospital General de Zona N 0. 2. de San Luis Potosí. Instituto Mexicano del Seguro Social. Unidad de Epidemiología Clínica. Facultad de Medicina. Universidad Autónoma de San Luis Potosí, San Luis Potosí, México.

Fecha de recibido: 21 de agosto de 2002 - Fecha de aprobado: 6 de mayo de 2003

Solicitud de sobretiros: D r. Juan Pablo Castanedo Cázares. Unidad de Fotobiología. Departamento de Dermatología. Facultad de Medicina. Universidad Autónoma de San Luis Potosí. Venustiano Carranza 2405. Zona Universitaria, 78210. San Luis Potosí, México.

Correo electrónico:castananju@ yahoo.com 
mes de exposición, mediante la fórmula $587.20+438.45$ (género)+500.16(mes)-49.65(mes²). El resultado muestra mayores dosis de radiación de marzo a septiembre. Conclusiones. Proponemos un marco teórico para la creación de políticas dirigidas a proteger a los alumnos de la sobrexposición solar durante su asistencia a la escuela en México. Se justifica la implantación de medidas preventivas durante al menos el periodo de mayor exposición (marzo a junio), ya que en este lapso se recibe $51 \%$ de la dosis anual de radiación ultravio leta. Si se evitara la exposición de $8 \mathrm{~min} /$ día se podrían reducir $39495 \mathrm{~J} / \mathrm{m}^{2}$, dosis que actualmente se acumula en dos años. El texto completo en inglés de este artículo está disponible en: http://www.insp.mx/salud/ index.html

Palabras clave:dosis; rayos ultravioleta; niño; adolescencia; México radiation doses between March and September. Conclusions. A theoretical framework is advanced to formulate policies aimed at protecting children in Mexican schools from solar overexposure. Implementing prevention measures at least during the months of greatest exposure (March to June) is in order, since $51 \%$ of the annual ultraviolet radiation dose is received in this period. By avoiding exposure 8 minutes daily, the radiation dose could be decreased in $39495 \mathrm{~J} / \mathrm{m}^{2}$, which is the dose accumulated in two years. The English version of this paper is available at: http:// www.insp.mx/salud/index.html

Key words: doses; ultraviolet rays; child adolescence; Mexico
$\mathrm{E}$ n el transcurso de su vida escolar los alumnos que acuden a los planteles educativos se exponen a la radiación del sol durante tiempos de receso, actos cívicos, actividades deportivas, y el regreso a casa. Esta radiación está compuesta por luz visible, infrarroja y ultravioleta (UV)..$^{1} \mathrm{El} 80 \%$ de los efectos indeseables que origina la exposición solar se debe al espectro comprendido entre los 290 y $320 \mathrm{~nm}$ de longitud (UVB). ${ }^{2}$ Cuantificar esta exposición es esencial pues existe una relación directa entre su magnitud y el desarrollo de neoplasias, ${ }^{3,4}$ mutaciones, ${ }^{5}$ fotoenvejecimiento, ${ }^{6}$ inmunosupresión ${ }^{7}$ y cataratas. ${ }^{8}$

Aunque estas lesiones se manifiestan durante la vida adulta, $80 \%$ del daño se establece durante la infancia y la adolescencia. ${ }^{9,10}$ Debido a esta relación causal, en diversas partes del mundo se han establecido campañas orientadas a reducir su impacto sobre poblaciones susceptibles. ${ }^{11-14}$ Por su localización geográfica, México es un país que recibe dosis elevadas de radiación UV, la mayor parte del año. En San Luis Potosí, como en el resto de nuestro territorio, la asistencia a la escuela es una actividad que se realiza durante las horas de mayor irradiación solar del día. Considerando estos antecedentes, los objetivos del presente estudio fueron: a) estimar la cantidad de radiación UV recibida por una población estudiantil de la ciudad de San Luis Potosí, durante su asistencia a la escuela primaria, secundaria y preparatoria; b) identificar diferencias entre los distintos grupos escolares, géneros y meses de exposición, y c) proponer medidas para reducir la radiación UV incidente sobre estos grupos.

\section{Material y métodos}

Medición de radiación ultravioleta. La determinación de los niveles de radiación UV se llevó a cabo en San Luis Potosí, capital del estado del mismo nombre, localizada a $22^{\circ} 09^{\prime}$ de latitud norte, $100^{\circ} 58^{\prime}$ longitud oeste y 1 877 metros de altitud. Con el fin de distinguir variaciones estacionales las lecturas se tomaron de mayo de 2001 a abril de 2002. Para llevar a cabo el estudio se estableció como día "representativo mensual" al promedio de siete determinaciones hechas a mediados de cada mes (días 14 al 20). La intensidad de la radiación UV se determinó mediante radiómetros calibrados a $297 \mathrm{~nm}$ (UVB) y $357 \mathrm{~nm}$ (UVA), (modelos 400-C y 500-C. National Biological Corp. Twinsburg, Ohio). Las mediciones se hicieron a intervalos horarios durante un lapso que recibe $75 \%$ de la irradiación solar ambiental diaria (por ejemplo de 9:00 a 15:00 hrs). ${ }^{15} \mathrm{El}$ sensor se orientó hacia el origen y a su alrededor se colocó un tubo oscuro de $10 \mathrm{~cm}$ de longitud, para evitar la dispersión y la reflectancia dentro del patio principal de las escuelas participantes. ${ }^{16} \mathrm{La}$ intensidad se midió en microwatts por centímetro cuadrado $\left(\mathrm{mw} / \mathrm{cm}^{2}\right)$, y conforme a lo propuesto por la Comisión Internacional sobre Iluminación (CIE); la dosis fue integrada al espectro de acción eritematógeno en joules por metro cuadrado $\left(\mathrm{J} / \mathrm{m}^{2}\right)$ para cada uno de los intervalos horarios. ${ }^{17-19}$

Estimación de la dosis ultravioleta. De forma aleatoria se formó un grupo de 80 alumnos pertenecientes a tres escuelas públicas, y una particular para determinar el 
tiempo de exposición solar durante su asistencia a estas instituciones. En el transcurso de un calendario escolar profesores voluntarios adiestrados monitorearon las actividades de estos escolares de forma simultánea y dentro del lapso escogido para elaborar la medición ambiental de radiación UV. El monitoreo se efectuó una vez al mes para cada uno de ellos. Mediante bitácora y cronómetro se registró, por edad y género, cualquier exposición solar desde su ingreso hasta su salida y regreso a casa. Se formaron cuatro grupos de 20 integrantes (10 hombres y 10 mujeres): 7 a 9 años de edad ( $1^{\text {er }}$ a $3^{\text {er }}$ grado primaria), 10 a 12 años de edad ( $4^{\circ}$ a $6^{\circ}$ grado primaria), 13 a 15 años de edad (secundaria) y 16 a 19 años de edad (preparatoria). El $90 \%$ de los alumnos $(n=72)$ fueron de piel morena (fototipos IV-V), y sólo $10 \%(n=8)$ de piel blanca (fototipo III)..$^{15}$ El tiempo de exposición solar por alumno fue registrado en minutos para cada uno de los intervalos monitoreados. Ninguna de estas escuelas disponía de explanadas o gimnasios techados para la práctica de actividades fuera del aula. La exposición solar fuera del horario de la escuela, así como la estancia en exteriores, pero bajo la sombra de árboles o edificios, no fue contabilizada. La dosis personal de UV diaria se consideró como 30\% de la radiación ambiental existente, pues su proporción incide sobre la cara de un individuo. ${ }^{20-23}$ La radiación ambiental que proporcionó la fracción para el cálculo fue el promedio de las siete determinaciones ambientales a mitad de cada mes (día representativo mensual). La dosis mensual se obtuvo multiplicando el promedio diario estimado por el número de días de clase hábiles al mes, y la anual, integrando la suma de todas éstas.

Análisis estadístico. Las variables se sometieron a análisis descriptivo univariado.

Posibles diferencias entre géneros se evaluaron mediante la prueba comparativa de KolmogorovSmirnof. La prueba de Kruskal-Wallis se empleó para evaluar las variaciones mensuales y por grupos, puesto que los residuos no muestran distribución normal. Posteriormente, por medio del uso de las técnicas estadísticas de modelos lineales generalizados (GLM, por sus siglas en inglés), y de la calificación por el índice de Akaike (AIC, por sus siglas en inglés) con sistema de búsqueda escalonada, se determinó el mejor modelo de regresión múltiple, considerando la dosis de exposición como variable de salida, y las variables género, grupo y mes como predictores. El análisis fue realizado con el programa estadístico R versión. 1.5.1. ${ }^{24}$

\section{Resultados}

Durante el horario de asistencia a la escuela matutina la dosis terrestre diaria de radiación UV alcanzó un máximo de $5005 \mathrm{~J} / \mathrm{m}^{2}$ en abril, y un mínimo de 2202 $\mathrm{J} / \mathrm{m}^{2}$ en el mes de diciembre (cuadro I). Aunque los niveles de radiación se registraron durante julio y agosto el monitoreo de los alumnos se vio interrumpido por el asueto de verano. El total de minutos laborados durante un día normal de asistencia a estas escuelas fue de 320 minutos en la primaria, 420 en secundaria, y 450 en preparatoria. En este lapso, 80\% del tiempo de exposición solar ocurre entre las 10:00 y las 13:00 hrs. Esto se debe, principalmente, a actividades hechas en el receso de descanso, en las clases de educación física, y a la salida de la escuela. Para efectuar lo anterior los intervalos sugeridos por el programa de estudios fueron de aproximadamente 60 a 70 $\mathrm{min} /$ día en primaria, 50 a $60 \mathrm{~min} /$ día en secundaria y de 40 a $50 \mathrm{~min} /$ día en preparatoria. Por lo tanto, la dosis potencialmente máxima de radiación UV durante una mañana de clase corresponde entre 18 a 21\%, 12 a $14 \%$ y 8 a $12 \%$ de la ambiental existente para cada nivel escolar, respectivamente.

\section{Cuadro I \\ Variación anUal en la dosis ambiental DE RADIACIÓN ULTRAVIOLETA POR INTERVALOS HORARIOS EN UN Día DE ASISTENCIA A LA ESCUELA.* San Luis Potosí, México, 2001-2002}

Hora $\begin{array}{lllllll}09: 00 & 10: 00 & 11: 00 & 12: 00 & 13: 00 & 14: 00 & 15: 00\end{array}$

\begin{tabular}{lllllllll} 
Enero & 277 & 335 & 404 & 425 & 400 & 363 & 310 & 2514 \\
\hline Febrero & 338 & 408 & 493 & 518 & 488 & 443 & 378 & 3066 \\
\hline Marzo & 503 & 607 & 733 & 770 & 725 & 725 & 562 & 4625 \\
\hline A bril & 385 & 581 & 701 & 847 & 889 & 838 & 761 & 5002 \\
\hline Mayo & 373 & 564 & 680 & 821 & 863 & 813 & 738 & 4852 \\
\hline Junio & 356 & 538 & 649 & 783 & 823 & 775 & 704 & 4628 \\
\hline Julio & 350 & 529 & 638 & 771 & 810 & 763 & 693 & 4554 \\
\hline A gosto & 321 & 486 & 586 & 708 & 743 & 700 & 636 & 4180 \\
\hline Septiembre & 293 & 442 & 534 & 644 & 677 & 638 & 579 & 3807 \\
\hline O ctubre & 258 & 390 & 471 & 565 & 598 & 565 & 511 & 3358 \\
\hline Noviembre & 286 & 345 & 417 & 438 & 413 & 375 & 320 & 2594 \\
\hline Diciembre & 243 & 293 & 354 & 371 & 348 & 320 & 271 & 2200
\end{tabular}

* Las mediciones se realizaron en la ciudad de San Luis Potosí a 22 09 de latitud norte, $100 \cong 58^{\prime}$ longitud oeste y 1877 metros de altitud de mayo de 2001 a abril de 2002. El horario considera el cambio de verano en abril, y otoño en octubre 


\section{Cuadro II \\ Dosis de radiación mensual ultravioleta durante el horario matutino por edad y SEXo. San Luis Potosí, México, 2001-2002}

\begin{tabular}{|c|c|c|c|c|c|c|c|c|c|c|}
\hline & & Mujer & $(n=40)$ & & & & $\mathrm{Hom}$ & $5(n=40)$ & & \\
\hline & $7-9$ & $10-12$ & $13-15$ & $16-19$ & Promedio & $7-9$ & $10-12$ & $13-15$ & $16-19$ & Promedio \\
\hline Enero & 1173 & 1081 & 1058 & 1012 & 1081 & 1426 & 1334 & 1380 & 1495 & 1408 \\
\hline Febrero & 1302 & 1218 & 1176 & 1060 & 1189 & 1596 & 1491 & 1554 & 1580 & 1555 \\
\hline Marzo & 1953 & 1806 & 1785 & 1680 & 1806 & 2373 & 2226 & 2310 & 2499 & 2352 \\
\hline Abril & 1995 & 1848 & 1827 & 1764 & 1858 & 2226 & 2268 & 2352 & 2604 & 2362 \\
\hline Mayo & 2116 & 1955 & 1955 & 1863 & 1972 & 2645 & 2415 & 2484 & 2760 & 2576 \\
\hline Junio & 1848 & 1701 & 1701 & 1617 & 1716 & 2352 & 2100 & 2163 & 2415 & 2257 \\
\hline Septiembre & 1440 & 1340 & 1320 & 1280 & 1354 & 2140 & 1640 & 1700 & 1880 & 1840 \\
\hline 0 ctubre & 1449 & 1357 & 1334 & 1288 & 1357 & 1771 & 1579 & 1725 & 1909 & 1771 \\
\hline Noviembre & 1166 & 1078 & 1034 & 990 & 1067 & 1408 & 1320 & 1364 & 1474 & 1391 \\
\hline Diciembre & 945 & 882 & 861 & 798 & 871 & 1155 & 1092 & 1113 & 1176 & 1134 \\
\hline Total & 15387 & 14266 & 14051 & 13352 & 14264 & 19092 & 17565 & 18145 & 19792 & 18648 \\
\hline
\end{tabular}

La dosis anual UV se obtuvo integrando la suma de las dosis mensuales. La dosis mensual representa la dosis diaria obtenida, multiplicada por el número de días hábiles del mes. La dosis personal UV diaria fue determinada considerando que la radiación incidente sobre la cara equivale a $30 \%$ de la ambiental existente. La radiacion UV ambiental representativa mensual fue considerada como el promedio de las mediciones obtenidas durante los días 14 a 20 de cada mes. La dosis ambiental se midió en el patio de sus respectivas escuelas, mediante radiometría terrestre, y de forma simultánea a la determinación del tiempo de exposición de los alumnos una vez por mes

En todos los grupos, la dosis personal de radiación fue menor en invierno, y máxima al finalizar primavera (cuadro II). La dosis mensual promedio para todos los grupos se incrementa $100 \%$ desde su nivel más bajo, en diciembre, hasta el más alto, en mayo $\left(1002 \mathrm{~J} / \mathrm{m}^{2}\right.$ vs. $2274 \mathrm{~J} / \mathrm{m}^{2}$ ).

La dosis de radiación mínima calculada correspondió al grupo de mujeres de 16 a 19 años de edad durante el mes de diciembre, con $798 \mathrm{~J} / \mathrm{m}^{2}$; y el máximo al grupo de hombres de 16 a 19 años de edad durante el mes de mayo, con $2760 \mathrm{~J} / \mathrm{m}^{2}$. Las mujeres, en conjunto, recibieron $23 \%$ menos radiación UV que los hombres $\left(14264 \mathrm{~J} / \mathrm{m}^{2}\right.$ vs $\left.18648 \mathrm{~J} / \mathrm{m}^{2}, p=0.003\right)$, lo cual es evidente en el análisis comparativo por géneros (figura 1). Esto se debió, principalmente, a que las primeras prefirieron permanecer jugando o platicando en la sombra; mientras que los segundos, jugar en exteriores durante los tiempos de receso.

No se encontraron diferencias en las dosis entre los distintos tipos de piel de los alumnos ni entre los grupos escolares $(p=0.8)$, pues éstas fueron similares. Sin embargo, al analizar la dosis de radiación por mes se hallaron diferencias significativas (Kruskall-Wallis $p>0.0001)$. Mediante el uso de modelos lineales generalizados (GLIM) y la búsqueda escalonada con la variable dosis como dependiente, y grupo, género y mes como predictores, se obtuvo el mejor modelo con una calificación de técnica escalonada y calificación del

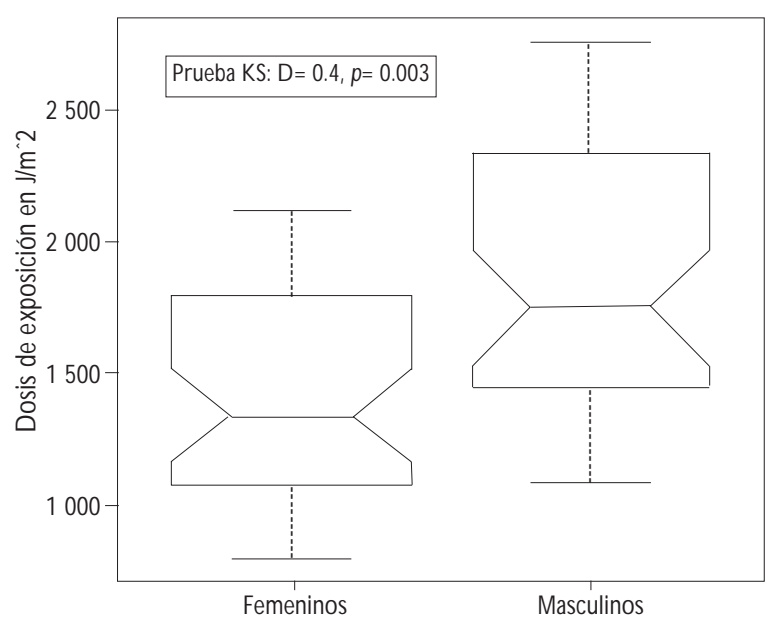

Figura 1. Gráfica de caja de las distribuciones de LA DOSIS DE RADIACIÓN EN JOULES POR METRO CUADRAdo POR SEXo. ANÁlisis comparativo realizado CON prueba de Kolmogorov-Smirnov. San Luis Potosí, MÉxICO, 2001-2002

índice de Akaike (AIC) de 1 083, que fue el siguiente: dosis de radiación $=587.20+438.45($ sexo $)+500.16$ (mes) - 49.65 (mes ${ }^{2}$ ).

salud pública de méxico / vol.45, no.6, noviembre-diciembre de 2003 


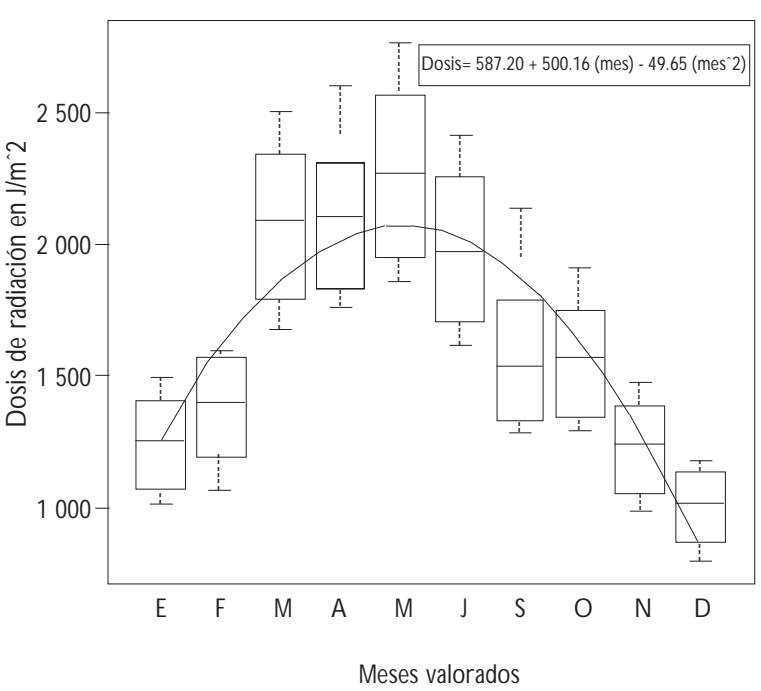

FiguRa 2.VARIación MENSUAL de LA EXPOSICIÓN A RADIACIÓN ULTRAVIOLETA EN JOUles POR METRO CUADRADO. San Luis Potosí, México, 2001-2002. Los diagramas DE CAJA REPRESENTAN LA EXPOSICIÓN MENSUAL DEL GRUPO Y SU DISTRIBUCIÓN POR CUARTILES. LA ECUACIÓN EN EL ÁNGULO SUPERIOR DERECHO EXPLICA LA LÍNEA PUNTEADA EN LA GRÁFICA, OBTENIDA POR GLIM CON TRANSFORMACIÓN CUADRÁTICA DE LA VARIABLE MES

Con las variables sexo y mes altamente significativas, esta ecuación permitió modelar la curva de exposición según el mes valorado, y se ajustó por género (figura 2). En la gráfica se hace evidente que en los meses de marzo, abril, mayo, junio y septiembre se reciben las mayores dosis del año, que son mayores en mayo.

\section{Discusión}

El cáncer del piel es la segunda neoplasia más frecuente en nuestro país. ${ }^{25}$ Debido a que la radiación UV es la responsable, en la mayoría de los casos, la implantación de acciones preventivas desde temprana edad podría ser uno de los mecanismos que permitieran establecer patrones de comportamiento saludables, que disminuyeran su incidencia en la vida adulta. ${ }^{26}$

La dosis de radiación UV depende, tanto del tiempo de exposición, como de la intensidad y distribución sobre diversos sitios anatómicos (por ejemplo, cara, manos, cuello). ${ }^{27,28}$ Dado que el estudio se basó en el cálculo y medición de estas variables, la dosis se estimó para la región facial, por ser el sitio invariablemente expuesto durante el año de observación.
Este es el primer informe en México que evalúa el patrón de exposición solar en un grupo de niños y adolescentes que asisten a la escuela durante un año lectivo. Se encontró que el tiempo de exposición solar corresponde, aproximadamente, a una cuarta parte de las actividades fuera del aula, y que no existen diferencias entre las dosis de los grupos de primaria, secundaria y preparatoria. Sin embargo, los hombres reciben mayores dosis que las mujeres en los tres niveles educativos, debido principalmente a que las primeras permanecen más tiempo en la sombra durante el receso.

Por medio de la observación de estos grupos hemos identificado un periodo crítico de cuatro meses (marzo a junio), en el que los alumnos de estas escuelas reciben $51 \%$ de la dosis anual de radiación UV. Por lo tanto, si se establecieran acciones preventivas durante al menos este periodo se lograrían reducciones significativas al concluir estos ciclos educativos. Si nos propusiéramos reducir en $20 \%$ la dosis UV diaria que en la actualidad reciben estos alumnos (aproximadamente ocho $\mathrm{min}$ /día), la exposición acumulada al cumplir los 20 años sería en promedio $39495 \mathrm{~J} / \mathrm{m}^{2}$ menor: es decir, la dosis recibida en el transcurso de dos años y cuatro meses. Si consideramos que las actividades escolares en nuestro país coinciden con las horas de mayor radiación solar del día, y que gran parte de la exposición personal se acumula antes de los 20 años de edad, ${ }^{9}$ el estudio cobra particular relevancia, ya que las dosis de radiación UV recibidas durante estos 12 años de escolaridad constituyen una fracción muy importante del total a acumularse durante la vida de una persona; lo anterior es susceptible de ser reducido.

Si intentáramos comparar las dosis de nuestra población con las de otras encontraríamos que existen múltiples factores como constitución genética, alimentación, cultura, educación, infraestructura, políticas escolares, clima, o localización geográfica, que pueden influir, tanto en el patrón de exposición solar, como en su respuesta biológica. ${ }^{22,23,29}$ Por lo tanto, es imposible establecer rangos ambientales de radiación UV seguros o confiables para grupos, como éste, de niños o adolescentes. La finalidad del trabajo fue cuantificar y proponer un marco teórico que favorezca la implantación de medidas dirigidas a reducir la exposición de nuestros escolares, considerando el daño que la radiación UV origina en los humanos. En México, pese a que el cáncer de piel constituye $14.7 \%$ del total de neoplasias malignas registradas, ${ }^{25}$ no contamos con programas de alerta pública. Como resultado, alumnos e instructores permanecen periodos prolongados bajo el sol, sin advertir sus consecuencias. Aunque se han realizado 
esfuerzos por advertir a la comunidad médica sobre los efectos de la radiación $\mathrm{UV}^{30}{ }^{30}$ es necesario promover acciones a mayor escala dirigidas a evitar la sobrexposición solar en la población escolar. Algunas implicarían dotar a las escuelas con la infraestructura necesaria para realizar sus actividades bajo techo. Sin embargo, a corto plazo creemos que se obtendrían mejores resultados educando a padres, alumnos y maestros sobre las consecuencias de la radiación solar, así como mediante la modificación de ciertas políticas escolares, como el cambio del horario en las actividades al aire libre, permitir el uso de gorros o sombreros, y además, promover la utilización de filtros solares apropiados. $^{31}$

\section{Agradecimientos}

Los autores agradecen la valiosa participación de los profesores Gilberto Santillán Ortiz, Blanca Lilia Cázares Ruiz, Laura Cázares Ruiz, Josué Aguirre Cázares, y Ramiro Osorio Mendoza en la realización de este estudio.

\section{Referencias}

1. Diffey BL.W hat is light? Photodermatol Photoimmunol Photomed 2002;18:68-74.

2. Cole CA, Forbes PD, D avies RE. An action spectrum for UV photocarcinogenesis. Photochem Photobiol 1986;36:187-191. 3. Koh HK, G eller AC, Miller DR, Grossbart TA, Lew RA. Prevention and early detection strategies for melanoma and skin cancer.Arch Dermatol 1996;132:436-443.

4. Ley RD, Reeves VE. Chemoprevention of ultraviolet radiation-induced skin cancer. Environ Health Perspect 1998;105:981-984.

5.W ikonkai N M, Brash DE. Ultraviolet radiation induced signature mutations in photocarcinogenesis. I Invest D ermatol Symp Proc 1999:46-49.

6.Yaar M, Gilchrest BA.A Aing and photoaging: Postulated mechanisms and effectors.J Invest Dermatol Symp Proc 1998:3:47-51.

7. N ishigori C, Yarosh D B, D onawho C, Kripke ML.The immune system in ultraviolet carcinogenesis. J Invest D ermatol Symp Proc 1996;1: 143-146.

8. Sliney D H. Epidemiological studies of sunlight and cataract:The critical factor of ultraviolet exposure geometry. 0 phtalmic Epidemiol 1994;1:107-119.

9. Stern RS,W einstein MC, Baker SG. Risk reduction for nonmelanoma skin cancer with childhood sunscreen use.Arch Dermatol 1986;122:537-545.
10. Parisi AV, Meldrum LR, W ong JC, Aitken J, Fleming RA. Effect of childhood and adolescent ultraviolet exposures on cumulative exposure in South East Q ueensland schools. Photodermatol Photoimmunol Photomed 2000;16:19-24.

11. Fork $H, W$ agner $R, W$ agner $K$. The Texas peer education sun awareness project for children: Primary prevention of malignant melanoma and nonmelanocytic skin cancers. Cutis 1992;50:363-364. 12. G lanz K, C hang L, Song V, Silverio R, Muneoka M. Skin cancer prevention for children, parents, and caregivers: A field test of Hawaii's SunSmart program. J Am Acad Dermatol 1998:38:413-417.

13. Smith BJ, Ferguson C, McKenzie J, Bauman A,Vita P. Impacts from repeated mass media campaigns to promote sun protection in Australia. Health Promot Int 2002;17:51-60.

14. Schulz EJ. Sun education in South Africa. C lin Dermatol 1998;16: 531-533.

15. Hönigsmann H. Erythema and pigmentation. Photodermatol Photoimmunol Photomed 2002;18:75-81.

16. Rigel DS, Rigel EG, Rigel AC. Effects of altitude and latitude on ambient UVB radiation. J Am Acad D ermatol 1999;40:114-116.

17. McKinlay AF, D iffey BL.A reference action spectrum for ultraviolet induced erythema in human skin. CIE J 1987; 6:17-22.

18. W ong CF, Toomey S, Fleming RA, Thomas BW. UV-B radiometry and dosimetry for solar measurements. Health Phys 1995; 68:175-184.

19. Bech-Thomsen N , Wulf HC. Carcinogenic potential of fluorescent UV tanning sources can be estimated using the CIE erythema action spectrum. Int J Radiat Biol 1993;64:445-450.

20. Leach JF, McLeod VE, Pingstone AR, D avis A, D eane GH. Mesurement of the ultraviolet doses received by office workers. Clin Exp Dermatol 1978;3:77-79.

21. Larko O, D iffey BL. N atural UV-B radiation received by people with outdoor, indoor and mixed occupations and UV-B treatment of psoriasis. Clin Exp Dermatol 1983;8:279-285.

22. Melville SK, Rosenthal FS, Luckmann R, Lew A. Q uantitative ultraviolet skin exposure in children during selected outdoor activities. Photodermatol Photoimmunol Photomed 1991;8:99-104.

23. Godar DE. UV doses of American children and adolescents. Photochem Photobiol 2001;74:787-793.

24. Ihaka R, Gentleman R. R:A language for data analysis and graphics. Comput Graph Stat 1991;5:299-314.

25.Secretaría de Salud. Registro histopatológico de neoplasias malignas. Compendio mortalidad, morbilidad. México, DF:SSA, 1999.

26. Centers for Disease Control and Prevention. Guidelines for school programs to prevent skin cancer. MMW R Morb Montal W kly Rep 2002;51(N O. RR-4):

27. Herlihy E, G ies PH, Roy CR, Jones M. Personal dosimetry of solar UV radiation for different outdoor activities. Photochem Photobiol 1994;60:288-294.

28. Diffey BL. Ultraviolet radiation and human health. Clin Dermatol 1998;16:83-89.

29. Diffey BL, Gibson CJ, Haylock R, McKinlay AF. O utdoor ultraviolet exposure of children and adolescents. Br J Dermatol 1996;134:

1030-1034.

30. C astanedo-C ázares JP, Moncada B. El índice ultravioleta. D ermatol Rev Mex 2001:45:284-286.

31. Buller D B, Geller AC, C antor M, Buller MK, Rosseel K, Benjes L et al. Sun protection policies and environmental features in US elementary schools. Arch Dermatol 2002;138:771-774. 